\title{
Parallel Preconditioners for Solutions of Dense Linear Systems with Tens of Millions of Unknowns
}

\author{
Tahir Malas ${ }^{1,2}$, Özgür Ergül ${ }^{1,2}$ and Levent Gürel ${ }^{1,2}$ \\ ${ }^{1}$ Department of Electrical and Electronics Engineering \\ ${ }^{2}$ Computational Electromagnetics Research Center (BiLCEM) \\ Bilkent University, TR-06800, Bilkent, Ankara, Turkey \\ E-mail: tmalas@ee.bilkent.edu.tr, ergul@ee.bilkent.edu.tr, lgurel@ bilkent.edu.tr
}

\begin{abstract}
We propose novel parallel preconditioning schemes for the iterative solution of integral equation methods. In particular, we try to improve convergence rate of the ill-conditioned linear systems formulated by the electric-field integral equation, which is the only integral-equation formulation for targets having open surfaces. For moderate-size problems, iterative solution of the near-field system enables much faster convergence compared to the widely used sparse approximate inverse preconditioner. For larger systems, we propose an approximation strategy to the multilevel fast multipole algorithm (MLFMA) to be used as a preconditioner. Our numerical experiments reveal that this scheme significantly outperforms other preconditioners. With the combined effort of effective preconditioners and an efficiently parallelized MLFMA, we are able to solve targets with tens of millions of unknowns, which are the largest problems ever reported in computational electromagnetics.
\end{abstract}

\section{INTRODUCTION}

Many real-life problems confronted in computational electromagnetics (CEM) necessitate the solution of linear systems with millions of unknowns. In this paper, we investigate parallel preconditioners for the iterative solution of such dense and large systems formulated by the electric-field integral equation (EFIE), which is notorious for producing difficultto-solve linear systems.

Thanks to the multilevel fast multipole algorithm (MLFMA) [1], the matrix-vector multiplication of a dense system produced by integral equation formulations is carried out in $\mathcal{O}(N \log N)$ computational complexity for a matrix of order $N$. Furthermore, recent attempts produced efficient parallelizations of the method [2], which is obligated by continuously increasing problem dimensions of CEM.

However, the success of the employed iterative method is mainly determined by the preconditioner [3]. For moderate size problems, the sparse approximate inverse (SAI) preconditioner is successful in preconditioning ill-conditioned EFIE systems. The setup of SAI requires some global array exchanges among processors, but using proper data structures and communication schemes, highly efficient implementations are possible. In a rowwise decomposition scheme, the application of the preconditioner is merely a sparse matrix-vector product, which requires only a gather type communication before the multiplication operation.

Particularly for larger problems, convergence could be faster if we could provide a better approximation to the the near-field 1-4244-1364-8/07/\$25.00 @2007 IEEE matrix or even the exact solution as a preconditioner. However, this approach is impractical whether we use denser SAI or exact LU factorization, because of the memory considerations. On the other hand, for preconditioning purposes, the Krylov subspace solvers merely require the solution of a linear system to a given vector. This solution can be supplied by another iterative process provided that the Krylov subspace solver used for the system solution is flexible. Considering this possibility, we propose to use the iterative solution of the near-field system as a preconditioner for the original system, then use the fixed SAI preconditioner as a preconditioner to the nearfield system. We call this preconditioning scheme NF/SAI. The effectiveness of the available preconditioner (i.e., SAI) is highly increased with this inner-outer solution scheme [4].

When we have the opportunity to use an iterative procedure for the preconditioning operation, we can use also MLFMA for the inner solver to have stronger preconditioners compared to NF/SAI. Since the inner solver is used merely for preconditioning, we don't need a matrix-vector multiplication that is as accurate as MLFMA. For this purpose, we develop an approximate MLFMA (AMLFMA), which performs a much faster matrix-vector multiplication with some relative error compared to (full) MLFMA. Hence, the system matrix whose matrix-vector multiplication is performed via AMLFMA is used as a preconditioner. By taking into account the far-field elements wisely, AMLFMA preconditioner proves to be much more effective compared to the near-field preconditioners. Hence, in modest durations, we have been able to solve largest EFIE systems reported to the best of our knowledge.

In the next section, we give a brief summary of integralequation methods and MLFMA for the sake of completeness. Then, we detail the aforementioned preconditioners and present numerical experiments performed to test them. Finally, we conclude by discussing the pros and cons of the three preconditioners.

\section{Integral EQuation Methods AND MLFMA}

EFIE is the mandatory choice among the integral equations for the geometries involving open geometries. It is formed by a physical boundary condition, which states that total tangential electric-field vanishes on a conducting surface. With 
this condition, EFIE can be expressed as

$$
\hat{\boldsymbol{t}} \cdot \int_{S^{\prime}} \mathrm{d} \mathbf{r}^{\prime} \overline{\boldsymbol{G}}\left(\mathbf{r}, \mathbf{r}^{\prime}\right) \cdot \boldsymbol{J}\left(\mathbf{r}^{\prime}\right)=\frac{i}{k \eta} \hat{\boldsymbol{t}} \cdot \boldsymbol{E}^{i n c}(\mathbf{r}),
$$

where $\boldsymbol{E}^{i n c}$ represents the incident electric-field, $S^{\prime}$ is the surface of the object, $\hat{\boldsymbol{t}}$ is any tangential unit vector on $S^{\prime}$, $\boldsymbol{J}\left(\mathbf{r}^{\prime}\right)$ is the unknown induced current residing on the surface, $\bar{G}\left(\mathbf{r}, \mathbf{r}^{\prime}\right)$ is the dyadic Green's function.

Upon the discretization of Equation 1 by the method of moments, we end up with a dense linear system. The surface of the objects are in general meshed with 1/10th of the wavelength for accuracy. Hence, for high frequencies where the scatterer or the radiator sizes become large in terms of the wavelength, the system matrix becomes also large.

When iterative methods are used to solve such systems, they can at best provide $\mathcal{O}\left(N^{2}\right)$ complexity. This is prohibitive for large problems. Hence, the solutions of such problems is viable only with fast methods such as MLFMA, which drops the complexity of the dense matrix-vector multiplication to $\mathcal{O}(N \log N)$.

MLFMA is proposed as a multilevel extension of the single level fast multipole method. In order to perform interactions between the basis and testing functions in a group-by-group manner, the whole geometry is placed into a cube and it is recursively divided into smaller ones until the smallest cubes contain only a few basis functions. During the partitioning, if any of the cubes becomes empty, recursion stops there. MLFMA replaces element-to-element interactions with cluster-to-cluster interactions in a multilevel scheme. This computational scheme relies on the factorization of the Green's function, which is valid only for basis and testing functions that are far from each other. In the lowest level, interactions between the near-field clusters are computed directly and stored in the sparse matrix $\overline{\boldsymbol{A}}^{\mathrm{NF}}$. Interactions among the farfield clusters are computed approximately but with controllable error. For this purpose, the radiated fields of each cluster are aggregated at the centers of the clusters. Then, for each pair of far-field clusters whose parents are near to each other, cluster-to-cluster interaction is computed via a translation. Finally, after the translations, the matrix-vector multiplication is completed by disaggregating the incoming fields to the centers of the testing clusters and onto the testing functions.

\section{Preconditioning the Electric-Field Integral EQUATION}

Treating the matrix elements corresponding to near- and far-field interactions in a different way, MLFMA defines a splitting of the system matrix as

$$
\overline{\boldsymbol{A}}=\overline{\boldsymbol{A}}^{\mathrm{NF}}+\overline{\boldsymbol{A}}^{\mathrm{FF}},
$$

where $\overline{\boldsymbol{A}}^{\mathrm{NF}}$ denotes the sparse matrix that corresponds to nearfield interactions and $\overline{\boldsymbol{A}}^{\mathrm{FF}}$ denotes the matrix that corresponds to far-field interactions. Since $\overline{\boldsymbol{A}}^{\mathrm{FF}}$ is not readily available, it is customary to construct preconditioners from $\overline{\boldsymbol{A}}^{\mathrm{NF}}$ assuming it to be a good approximation to $\overline{\boldsymbol{A}}$. Typical members of this class are the incomplete factorization methods, which are based on eliminating some of the entries during the LU factorization [5]. After decomposing the near-field matrix in the form of $\overline{\boldsymbol{A}}^{\mathrm{NF}} \approx \overline{\boldsymbol{L}} \cdot \overline{\boldsymbol{U}}$, preconditioning operation is performed in each step by solving $\overline{\boldsymbol{L}} \cdot \overline{\boldsymbol{U}} \cdot \boldsymbol{v}=\boldsymbol{w}$, where $\overline{\boldsymbol{L}}$ and $\overline{\boldsymbol{U}}$ are the incomplete factors. On the other hand, a sparse approximate inverse $\overline{\boldsymbol{M}}$ directly approximates the inverse of the matrix and application of the preconditioner is performed simply with the sparse-matrix vector multiplication $\boldsymbol{v}=\overline{\boldsymbol{M}} \cdot \boldsymbol{w}$. The backward and forward substitutions required in the incomplete factorization methods are inherently sequential; hence for parallel applications approximate inverse type preconditioners are preferred.

There are various types of SAI preconditioners. Among them, the one that is based on Frobenius norm minimization is successfully used in CEM problems [6], [7]. After determining the sparsity pattern of the preconditioner, the approximate inverse of the near-field matrix is performed by minimizing

$$
\left\|\overline{\boldsymbol{I}}-\overline{\boldsymbol{M}} \cdot \overline{\boldsymbol{A}}^{\mathrm{NF}}\right\|_{F} .
$$

Minimization can be performed independently for each row by using the identity

$$
\left\|\overline{\boldsymbol{I}}-\overline{\boldsymbol{M}} \cdot \overline{\boldsymbol{A}}^{\mathrm{NF}}\right\|_{F}^{2}=\sum_{i=1}^{N}\left\|\boldsymbol{e}_{\boldsymbol{i}}-\boldsymbol{m}_{\boldsymbol{i}} \cdot \overline{\boldsymbol{A}}^{\mathrm{NF}}\right\|_{2}^{2},
$$

where $\boldsymbol{e}_{\boldsymbol{i}}$ is the $i$ th unit row vector and $\boldsymbol{m}_{\boldsymbol{i}}$ is the $i$ th row of the preconditioner. The nonzero pattern of $\bar{M}$ is fixed in advance. Usually, the pattern of $\overline{\boldsymbol{A}}^{\mathrm{NF}}$ is preferred but filtering may be adequate sometimes.

However, it is known that SAI is not as successful as ILU when we use the same amount of memory [8]. In Table II, we confirm this conviction by comparing SAI with the exact solution of the near-field matrix, which we call NF-LU. "Iter" denotes the number of iterations and "Soln" denotes the solution time in seconds. The stopping tolerance of GMRES is set to $10^{-6}$. The experiments are carried out on a shared memory system that consists of 8 dual-core AMD processors. The geometry information is detailed in Figure 1 and Table I. Though ILUT produces very close iteration counts to those of NF-LU [9], SAI deviates from this optimum behavior, as the number of unknowns increase. For a remedy, increasing the density of the preconditioner is undesirable because of possible high setup time and memory considerations.

On the other hand, since SAI is a good approximation to the inverse of the near-field matrix, a fast iterative solution of the system involving near-field matrix can be obtained and used as a preconditioner. This approach produces a nested implementation of iterative solvers. In the outer solver that solves the original system, we use FGMRES, a flexible version of GMRES, which allows the preconditioner to change from iteration to iteration. Then, the preconditioner of this solver can be another preconditioned Krylov subspace solver which is called the inner solver. We solve the near-field system in the inner solver, using SAI as the fixed preconditioner. We illustrate this preconditioning scheme in Figure 2. 


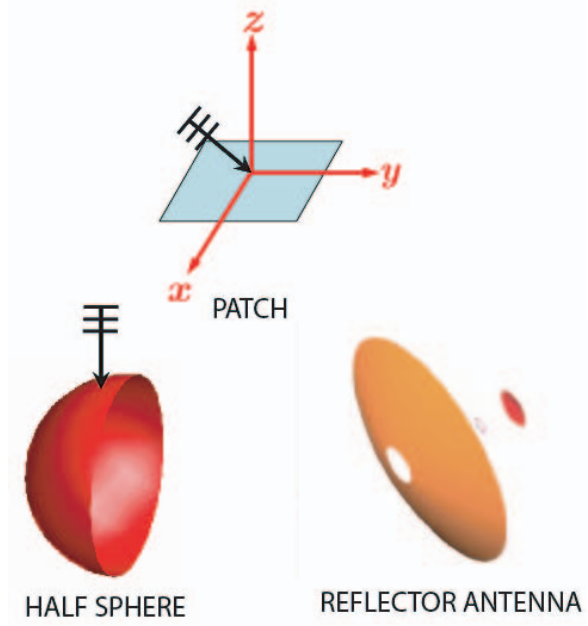

Fig. 1. Geometries used in the numerical experiments.

TABLE I

INFORMATION ABOUT THE OPEN GEOMETRIES. THE ABBREVIATION FOR THE PATCH IS "P", THE HALF SPHERE IS "HS", AND THE REFLECTOR ANTENNA IS "RA". "SIZE" DENOTES THE LENGTH OF THE MAXIMUM DIMENSION IN TERMS OF THE WAVELENGTH.

\begin{tabular}{|c|r|r|r|}
\hline Problem & $\begin{array}{c}\text { Frequency } \\
(\mathrm{GHz})\end{array}$ & $\begin{array}{c}\text { Size } \\
(\lambda)\end{array}$ & \multicolumn{1}{c|}{$N$} \\
\hline \hline P1 & 6 & 6 & 12,249 \\
\hline P2 & 20 & 20 & 137,792 \\
\hline P3 & 96 & 96 & $3,164,544$ \\
\hline P4 & 192 & 192 & $12,662,016$ \\
\hline P5 & 256 & 256 & $21,965,824$ \\
\hline \hline HS1 & 2.31 & 4.6 & 9,911 \\
\hline HS2 & 7.89 & 15.8 & 116,596 \\
\hline HS3 & 36.96 & 73.6 & $2,554,736$ \\
\hline HS4 & 73.92 & 147.2 & $10,221,280$ \\
\hline \hline RA1 & 1 & 25 & 356,439 \\
\hline RA2 & 3.73 & 94 & $2,515,103$ \\
\hline
\end{tabular}

TABLE II

PERFORMANCE COMPARISON OF SAI AND NF/SAI PRECONDITIONERS THE DASH “-” INDICATES THAT THE SOLUTION CANNOT BE OBTAINED DUE TO MEMORY LIMITATIONS.

\begin{tabular}{|c|r|r|r|r|r|r|}
\hline $\begin{array}{c}\text { Geo- } \\
\text { metry }\end{array}$ & $\begin{array}{c}\text { NF-LU } \\
\text { Iter }\end{array}$ & \multicolumn{3}{|c|}{ Setup } & \multicolumn{1}{c|}{ Iter } & \multicolumn{2}{c|}{ Soln } & \multicolumn{2}{c|}{ Iter } & \multicolumn{1}{c|}{ Soln } \\
\hline \hline P1 & 26 & 4 & 44 & 12 & 29 & 9 \\
\hline P2 & 53 & 52 & 91 & 336 & 59 & 253 \\
\hline P3 & - & 275 & 253 & 7,621 & 165 & 5,387 \\
\hline \hline HS1 & 38 & 7 & 60 & 24 & 40 & 17 \\
\hline HS2 & 93 & 77 & 156 & 510 & 103 & 383 \\
\hline HS3 & - & 381 & 547 & 17,404 & 380 & 12,286 \\
\hline \hline RA1 & - & 952 & 125 & 878 & 71 & 646 \\
\hline
\end{tabular}

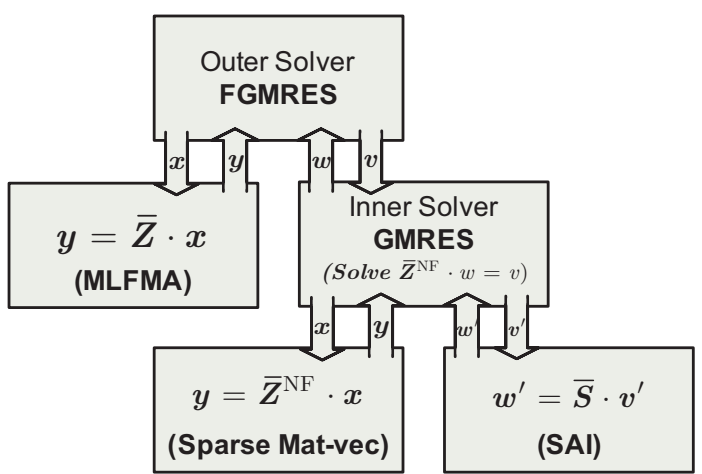

Fig. 2. Graphical representation of the inner-outer solution scheme.

Since the inner solver is used for preconditioning purposes, a rough solution can be adequate. Hence, we use GMRES as the inner solver since it provides a fast drop of the residual norm in the early iterations. For the stopping criteria of the inner solver, we conclude that only one order residual drop provides a very good preconditioner, which is attained with only a few iterations. The results presented in Table II reveals that such a crude solution of the near-system outperforms SAI and produce iteration counts which are very close to those of NF-LU.

However, when the size of the problem becomes very large, either convergence cannot be attained in a reasonable number of iterations or the available memory is exhausted by the no-restart GMRES. The lack of the effectiveness of SAI or NF/SAI stems from the fact that, as the problem size and the number of levels increase in MLFMA, the near-field matrix becomes too sparse. Hence, it does not carry enough information for preconditioning EFIE matrices, which are far from being diagonally dominant. For this purpose, we propose to use an approximate version of MLFMA for the inner solver. AMLFMA is obtained by systematically decreasing the truncation number of the translation function. We set the stopping tolerance of the inner solver to 0.1 as in the case of $\mathrm{NF} / \mathrm{SAI}$, but we let 10 iterations for the inner solver to reach this tolerance.

We first compare the AMLFMA preconditioner with SAI and NF/SAI in Fig. 3. Even though SAI and NF/SAI succeeds to converge with this problem, AMLFMA preconditioner decreases the solution time by $30 \%$ with respect to NF/SAI and $55 \%$ with respect to SAI. On the other hand, for the largest problems reported in Table III, convergence cannot be attained for three problems among four. These results are obtained on 32 processors of a cluster connected by Infiniband network. The stopping tolerance of GMRES is set to $10^{-6}$, except P5, for which the solution is achieved using $10^{-3}$ tolerance. With AMLFMA preconditioner, we succeed to solve largest P5 and HS4 problems in moderate iteration counts and solution times. In addition, we have been able to solve a reallife problem involving a very large reflector antenna, which is again unsolvable with other preconditioners.

Finally, we verify the solution of the largest patch problem 


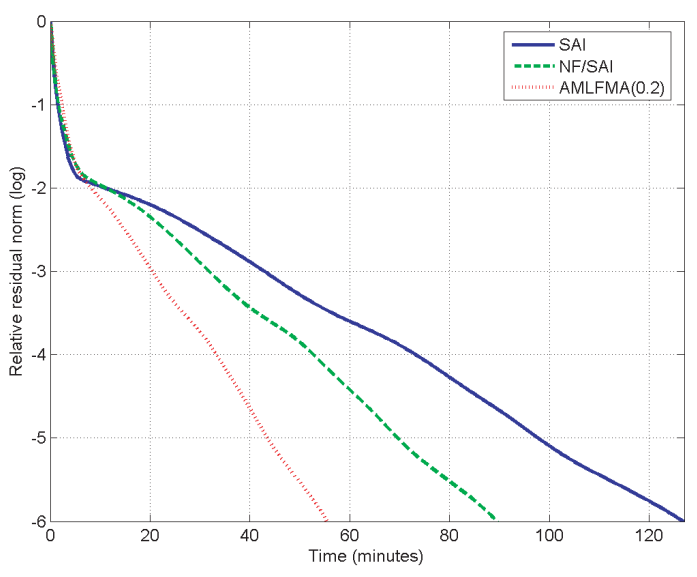

Fig. 3. Comparison of SAI, NF/SAI, and AMLFMA preconditioners for P3. Results are obtained on 32 processors of a cluster connected by Infiniband network.

TABLE III

COMPARISON OF SAI AND AMLFMA PRECONDITIONERS FOR THE LARGEST PROBLEMS IN TABLE I.

\begin{tabular}{|c|c|c|c|c|}
\hline \multirow{2}{*}{$\begin{array}{l}\text { Geo- } \\
\text { metry }\end{array}$} & \multicolumn{2}{|c|}{ SAI } & \multicolumn{2}{|c|}{ AMLFMA } \\
\hline & Iter & Soln & iter & Soln \\
\hline $\mathrm{P} 4$ & 275 & 33,557 & 53 & 16,184 \\
\hline P5 & - & - & 9 & 24,689 \\
\hline HS4 & $>1,000$ & - & 44 & 20,774 \\
\hline$\overline{\text { RA2 }}$ & $>1,000$ & - & 322 & 25,740 \\
\hline
\end{tabular}

by comparing it with a physical optics (PO) solution in Fig. 4. PO technique can provide very fast solutions, but the result is accurate for very large geometries and for specific locations. Since we illuminate patch from the direction $\left(\theta=45^{\circ}, \phi=\right.$ $0^{\circ}$ ), we expect accurate results from $\mathrm{PO}$ at specular reflection angle (which corresponds to $\left(\theta=45^{\circ}, \phi=180^{\circ}\right)$ ) and forward scattering (which corresponds to $\left(\theta=135^{\circ}, \phi=180^{\circ}\right)$ ). Hence, the accuracy of the MLFMA solution is verified with a perfect agreement between the two methods at these points.

\section{CONCLUSION}

In this work we summarized our efforts for preconditioning linear systems formulated by EFIE. The widely used SAI can be efficiently parallelized and yields a preconditioner with $\mathcal{O}(N)$ complexity. On the other hand, it cannot use the information provided by the near-field system efficiently. Hence, we propose to use the iterative solution of the nearfield system as a preconditioner. This scheme increased the effectiveness of the SAI preconditioner.

On the other hand, for very large problems, the near-field system itself becomes a too crude approximation to dense system matrix. Therefore, preconditioners that are built from the near-field interactions cannot be effective. Considering this fact, we developed AMLFMA preconditioner. Taking into account the far-field interactions as well as near-field interactions, AMLFMA preconditioner succeeds to solve ultra large systems in reasonable solution times. In particular, we

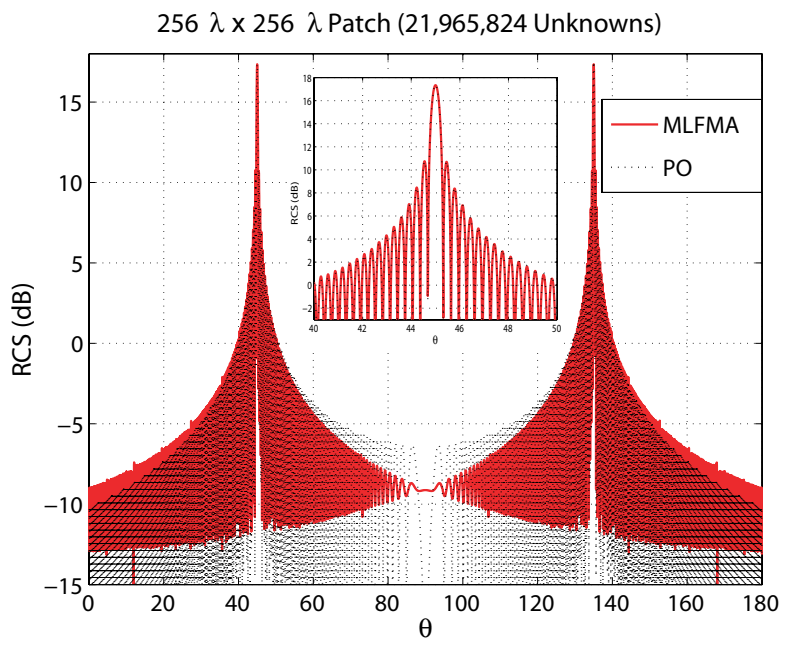

Fig. 4. Comparison the MLFMA solution with the PO solution for the $256 \lambda$ patch.

are able to solve a patch problem including approximately 22 millions of unknowns. We verify the accuracy of the solution by comparing the problem with its PO solution. To the best of our knowledge, this is the largest EFIE problem ever solved.

\section{ACKNOWLEDGMENT}

This work was supported by the Scientific and Technical Research Council of Turkey (TUBITAK) under Research Grant 105E172, by the Turkish Academy of Sciences in the framework of the Young Scientist Award Program (LG/TUBAGEBIP/2002-1-12), and by contracts from ASELSAN and SSM. Computer time was provided in part by a generous allocation from Intel Corporation.

\section{REFERENCES}

[1] W. C. Chew, J.-M. Jin, E. Michielssen, and J. Song, Eds., Fast and Efficient Algorithms in Computational Electromagnetics. Norwood, MA, USA: Artech House, Inc., 2001.

[2] S. Velamparambil, J. Song, and W. C. Chew, "On the parallelization of electrodynamic multilevel fast multipole method on distributed memory computers," in IWIA'99: Proceedings of the 1999 International Workshop on Innovative Architecture. Washington, DC, USA: IEEE Computer Society, 1999, p. 3.

[3] Y. Saad, Iterative Methods for Sparse Linear Systems, 2nd ed. Philadelphia, USA: SIAM, 2003.

[4] V. Simoncini and D. B. Szyld, "Flexible inner-outer krylov subspace methods," SIAM J. Numer. Anal., vol. 40, no. 6, pp. 2219-2239, 2002.

[5] M. Benzi, "Preconditioning techniques for large linear systems: a survey," J. Comput. Phys., vol. 182, no. 2, pp. 418-477, 2002.

[6] B. Carpentieri, I. S. Duff, L. Giraud, and G. Sylvand, "Combining fast multipole techniques and an approximate inverse preconditioner for large electromagnetism calculations," SIAM J. Sci. Comput., vol. 27, no. 3, pp. 774-792, 2005.

[7] J. Lee, J. Zhang, and C.-C. Lu, "Sparse inverse preconditioning of multilevel fast multipole algorithm for hybrid integral equations in electromagnetics," IEEE Trans. Antennas and Propagation, vol. 52, no. 9, pp. 158-175, 2004.

[8] M. Benzi and M. Tuma, "A comparative study of sparse approximate inverse preconditioners," Applied Numerical Mathematics: Transactions of IMACS, vol. 30, no. 2-3, pp. 305-340, 1999.

[9] T. Malas and L. Gürel, "Incomplete LU preconditioning with the multilevel fast multipole algorithm for electromagnetic scattering," SIAM J. Sci. Comput., vol. 29, no. 4, pp. 1476-1494, 2007, DOI: 10.1137/060659107. 